\title{
Common Fixed Points for Nonlinear Quasi-Contractions of Ćirić Type
}

\author{
Fei He \\ School of Mathematical Sciences, Inner Mongolia University, Hohhot 010021, China \\ Correspondence should be addressed to Fei He; hefei@imu.edu.cn
}

Received 25 March 2014; Revised 28 May 2014; Accepted 11 June 2014; Published 3 July 2014

Academic Editor: Geraldo Botelho

Copyright (C) 2014 Fei He. This is an open access article distributed under the Creative Commons Attribution License, which permits unrestricted use, distribution, and reproduction in any medium, provided the original work is properly cited.

We establish common fixed points theorems for two self-mappings satisfying a nonlinear contractive condition of Ćirić type with a Q-function. Furthermore, using the scalarization method, we deduce some results of common fixed point in tvs-cone metric spaces with a $c$-distance. As application, we give a positive answer to the question of Ćirić et al. posed in 2012. Our results extend and generalize many recent results.

\section{Introduction and Preliminaries}

In 1996, Kada et al. [1] for the first time introduced and studied the concept of $w$-distances on a metric space. They gave examples of $w$-distance and improved Caristi's fixed point theorem, Ekeland's variational principle, and Takahashi's nonconvex minimization theorem. Later, Al-Homidan et al. [2] introduced the concept of $Q$-functions which generalized the concept of $w$-distances, and they established a generalized Ekeland-type variational principle with a $Q$-function.

Definition 1. Let $X$ be a metric space with metric $d$. Then a function $q: X \times X \rightarrow[0, \infty)$ is called a $Q$-function on $X$ if the following are satisfied:

(q1) $q(x, z) \leq q(x, y)+q(y, z)$, for any $x, y, z \in X$;

(q2) if $x \in X$ and $\left\{y_{n}\right\}_{n \in \mathbf{N}}$ is a sequence in $X$ such that it converges to a point $y$ and $q\left(x, y_{n}\right) \leq M$ for some $M=M(x)>0$, then $q(x, y) \leq M$;

(q3) for any $\varepsilon>0$, there exists $\delta>0$ such that $q(z, x) \leq \delta$ and $q(z, y) \leq \delta$ imply $d(x, y) \leq \varepsilon$.

If condition (q2) is replaced by the following stronger condition:

$\left(\mathrm{q}^{\prime}\right)$ for any $x \in X, q(x, \cdot): X \rightarrow[0, \infty)$ is lower semicontinuous, then $q$ is called a $w$-distance on $X$.
For some examples of $Q$-functions and $w$-distances, the reader can see $[1,2]$. The following lemma has been presented in $[1,2]$.

Lemma 2. Let $(X, d)$ be a metric space and let $q$ be a $Q$ function on $X$. Let $\left\{x_{n}\right\}$ and $\left\{y_{n}\right\}$ be sequences in $X$, let $\left\{\alpha_{n}\right\}$ and $\left\{\beta_{n}\right\}$ be sequences in $[0,+\infty)$ converging to 0 , and let $x, y, z \in X$. Then the following hold.

(i) If $q\left(x_{n}, y\right) \leq \alpha_{n}$ and $q\left(x_{n}, z\right) \leq \beta_{n}$ for any $n \in \mathbf{N}$, then $y=z$. In particular, if $q(x, y)=0$ and $q(x, z)=0$, then $y=z$.

(ii) If $q\left(x_{n}, y_{n}\right) \leq \alpha_{n}$ and $q\left(x_{n}, z\right) \leq \beta_{n}$ for any $n \in \mathbf{N}$, then $\left\{y_{n}\right\}$ converges to $z$.

(iii) If $q\left(x_{n}, x_{m}\right) \leq \alpha_{n}$ for any $n, m \in \mathbf{N}$ with $m>n$, then $\left\{x_{n}\right\}$ is a Cauchy sequence.

(iv) If $q\left(y, x_{n}\right) \leq \alpha_{n}$ for any $n \in \mathbf{N}$, then $\left\{x_{n}\right\}$ is a Cauchy sequence.

Ume [3] proved some fixed point theorems in a complete metric space using the concept of a $w$-distance and more general contractive mapping than quasi-contractive mapping. Ilic and Rakocevic [4] established some common fixed points results for maps on metric spaces with $w$-distance and generalized the results of Ume [3]. Recently, di Bari and Vetro [5] obtained common fixed points for maps satisfying a nonlinear contractive condition. As application, using the 
scalarization method of $\mathrm{Du}$ [6], they deduce a result of common fixed point in cone metric space. For more early results in cone metric spaces (or in $K$-metric spaces) one can consider Zabrejko [7] and the references therein.

In 2007, Huang and Zhang [8] reintroduced and studied the concept of cone metric spaces over a real Banach space which is a generalization of metric spaces, and they proved several fixed point theorems in cone metric spaces. Then, many authors have studied fixed point problems in cone metric spaces; see [9] for a survey of fixed point results in these spaces. Recently, Du [6] used the scalarization function and showed that many of the fixed point theorems in metric spaces and in a generalized cone metric space (i.e., a so-called tvs-cone metric space) are equivalent. For more scalarization methods on fixed point problems, see also [10-12].

In the following we suppose that $E$ is a real Hausdorff topological vector space (tvs for short) with the zero vector $\theta$. A nonempty subset $K$ of $E$ is called a convex cone if $K+K \subseteq K$ and $\lambda K \subseteq K$ for $\lambda \geq 0$. A convex cone $K$ is said to be pointed if $K \cap(-K)=\{\theta\}$. For a given cone $K \subseteq E$, we can define a partial ordering $\preceq_{K}$ with respect to $K$ by $x \preceq_{K} y$ if and only if $y-x \in K . x \prec_{K} y$ will stand for $x \unlhd_{K} y$ and $x \neq y$, while $x \ll y$ stand for $y-x \in \operatorname{int} K$, where int $K$ denotes the interior of $K$.

Throughout this paper, unless otherwise specified, we always assume that $E$ is a tvs, $K$ a proper, closed, and convex pointed cone in $E$ with int $K \neq \emptyset$, and $e \in \operatorname{int} K$. Following $[6,8]$, we give the following notion.

Definition 3. Let $X$ be a nonempty set. A function $p: X \times$ $X \rightarrow E$ is called a tvs-cone metric space if the following conditions hold:

(p1) $\theta \unlhd_{K} p(x, y)$ for all $x, y \in X$ and $p(x, y)=\theta$ if and only if $x=y$;

(p2) $p(x, y)=p(y, x)$ for all $x, y \in X$;

(p3) $p(x, z) \unlhd_{K} p(x, y)+p(y, z)$ for all $x, y, z \in X$.

Let $x \in X$ and $\left\{x_{n}\right\}$ be a sequence in $X$. Then, it is said that

(i) $\left\{x_{n}\right\}$ converges to $x$ if for every $c \in E$ with $\theta \ll c$ there exists a natural number $n_{0}$ such that $p\left(x_{n}, x\right) \ll c$ for all $n>n_{0}$; we denote it by $x_{n} \rightarrow x$ as $n \rightarrow \infty$;

(ii) $\left\{x_{n}\right\}$ is a Cauchy sequence if for every $c \in E$ with $\theta \ll c$ there exists a $n_{0}$ such that $p\left(x_{n}, x_{m}\right) \ll c$ for all $m, n>$ $n_{0}$;

(iii) $(X, p)$ is complete if every Cauchy sequence is convergent in $X$.

Recently, some generalized distance on cone metric spaces is introduced. Cho et al. [13] introduced the concept of a $c$-distance in cone metric spaces and proved some fixed point theorems in ordered cone metric spaces. Ćirić et al. [14] introduce the concept of a $w$-cone distance on tvs-cone metric spaces and proved various fixed point theorems for $w$-cone distance contraction mappings in tvs-cone metric spaces. Let us recall these concepts.
Definition 4. Let $(X, p)$ be a tvs-cone metric space. Then a function $h: X \times X \rightarrow E$ is called a $c$-distance on $X$ if the following conditions hold:

(c1) $\theta \unlhd_{K} h(x, y)$ for all $x, y \in X$;

(c2) $h(x, z) \unlhd_{K} h(x, y)+h(y, z)$ for all $x, y, z \in X$;

(c3) for each $x \in X$ and $n \geq 1$, if $h\left(x, y_{n}\right) \unlhd_{K} u$ for some $u=$ $u_{x} \in K$, then $h(x, y) \unlhd_{K} u$ whenever $\left\{y_{n}\right\}$ is a sequence in $X$ converging to a point $y \in X$;

(c4) for all $c \in E$ with $\theta \ll c$, there exists $\eta \in E$ with $\theta \ll \eta$ such that $h(z, x) \ll \eta$ and $h(z, y) \ll \eta$ imply $p(x, y) \ll c$.

If condition (c3) is replaced by the following stronger condition:

(c3') for any $x \in X, h(x, \cdot): X \rightarrow K$ is lower semicontinuous, then $h$ is called a $w$-cone distance on $X$.

Remark 5 (see [13]). (1) $h(x, y)=h(y, x)$ does not necessarily hold for all $x, y \in X$.

(2) $h(x, y)=\theta$ is not necessarily equivalent to $x=y$.

Ćirić et al. [14] posed a question as follows.

Question 1. Let $(X, p)$ be a complete tvs-cone metric space with $w$-cone distance $h$ on $X$. Suppose $T: X \rightarrow X$ such that, for some constant $k \in(0,1)$ and for every $x, y \in X$, there exists $u \in\{h(x, y), h(x, T x), h(y, T y), h(x, T y), h(y, T x)\}$, such that $h(T x, T y) \unlhd_{K} k u$. Does there exist a unique fixed point $u \in X$ of $T$, and $h(u, u)=\theta$ ?

In this paper, we establish common fixed points theorems for two self-mappings satisfying a nonlinear contractive condition of Cirić type with a $Q$-function, which generalize the result of di Bari and Vetro [5]. Furthermore, using the scalarization method, we deduce some results of common fixed points in tvs-cone metric spaces with a $c$-distance. As application, we give a positive answer to Question 1 and extend some recent results presented in $[15,16]$. In particular, we will see that the assumption that $T$ satisfies, for all $x, y \in$ $X$,

$$
\begin{gathered}
h(T y, T x) \unlhd_{K} A h(y, x)+B h(T x, x)+C h(T y, x) \\
+D h(T y, y)+E h(T x, y)
\end{gathered}
$$

can be completely removed in [15].

\section{The Scalarization Method}

First, we recall the concept of the nonlinear scalarization function, which is one of the most useful tools to solve problems in vector optimization, control theory, and so forth; see, for example, [17-20].

The function $\xi_{e}: E \rightarrow \mathbf{R}$ is defined as follows:

$$
\xi_{e}(y)=\inf \{r \in \mathbf{R}: y \in r e-K\}, \quad \forall y \in E .
$$

Lemma 6 (see [17]). For each $r \in \mathbf{R}$ and $y \in E$, the following statements are satisfied: 
(i) $\xi_{e}(y) \leq r \Leftrightarrow y \in r e-K \Leftrightarrow y \unlhd_{K} r e$;

(ii) $\xi_{e}(y)<r \Leftrightarrow y \in r e-\operatorname{int} K \Leftrightarrow y \ll r e$;

(iii) $\xi_{e}(y) \geq r \Leftrightarrow y \notin r e-\operatorname{int} K$;

(iv) $\xi_{e}(y)>r \Leftrightarrow y \notin r e-K$;

(v) $\xi_{e}(y)=r \Leftrightarrow y \in r e-(K \backslash \operatorname{int} K)$, in particular, $\xi_{e}(\alpha e)=$ $\alpha$, for all $\alpha \in \mathbf{R}$;

(vi) $\xi_{e}$ is positively homogeneous and continuous on $E$;

(vii) if $y_{1} \in y_{2}+K$, that is, $y_{2} \preceq_{K} y_{1}$, then $\xi_{e}\left(y_{2}\right) \leq \xi_{e}\left(y_{1}\right)$;

(viii) if $y_{1} \in y_{2}+$ int $K$, that is, $y_{2} \ll y_{1}$, then $\xi_{e}\left(y_{2}\right)<\xi_{e}\left(y_{1}\right)$;

(ix) $\xi_{e}\left(y_{1}+y_{2}\right) \preceq_{K} \xi_{e}\left(y_{1}\right)+\xi_{e}\left(y_{2}\right)$ for all $y_{1}, y_{2} \in E$.

$\mathrm{Du}[6]$ used the above scalarization function and obtained the following results.

Theorem 7 (see [6, Theorem 2.1]). Let $(X, p)$ be tvs-cone metric spaces. Then $d_{p}: X \times X \rightarrow[0, \infty)$ defined by $d_{p}:=\xi_{e} \circ p$ is a metric.

Theorem 8 (see [6, Theorem 2.2]). Let $(X, p)$ be a tvs-cone metric space, $x \in X$, and $\left\{x_{n}\right\}$ a sequence in $X$. Let $d_{p}$ be the same as in Theorem 7 . Then the following statements hold:

(i) $\left\{x_{n}\right\}$ converges to $x$ in $(X, p)$ if and only if $\left\{x_{n}\right\}$ converges to $x$ in $\left(X, d_{p}\right)$;

(ii) $\left\{x_{n}\right\}$ is a Cauchy sequence in $(X, p)$ if and only if $\left\{x_{n}\right\}$ is a Cauchy sequence in $\left(X, d_{p}\right)$;

(iii) $(X, p)$ is complete if and only if $\left(X, d_{p}\right)$ is complete.

Proof. Du has proven the necessity of (i) and (ii); see [6, Theorem 2.2]. As the proof of (ii) is similar to that of (i) and (iii) follows from (i) and (ii), it is enough to prove the sufficiency of (i).

Assume now that $x_{n} \stackrel{d_{p}}{\longrightarrow} x$ and $c \in E$ with $\theta \ll c$. Then $c \in$ int $K$ and consequently $c-$ int $K$ is a neighborhood of $\theta$. For $e \in E$, there is $\delta>0$ such that $\delta e \in c-$ int $K$; that is, $\delta e \ll c$. Since $d_{p}\left(x_{n}, x\right) \rightarrow 0$ as $n \rightarrow \infty$, there exists a positive integer $n_{0}$ such that

$$
\xi_{e} \circ p\left(x_{n}, x\right)=d_{p}\left(x_{n}, x\right)<\delta \quad \forall n>n_{0} .
$$

By (ii) of Lemma 6, we have $p\left(x_{n}, x\right) \ll \delta e$ for all $n>n_{0}$. From this and $\delta e \ll c$, we obtain

$$
p\left(x_{n}, x\right) \ll c \quad \forall n>n_{0} .
$$

Thus $\left\{x_{n}\right\}$ converges to $x$ in $(X, p)$.

Now, inspired by the scalarization method of $\mathrm{Du}[6]$, we give the following result.

Theorem 9. Let $(X, p)$ be a tvs-cone metric space and let $h$ be a c-distance on $X$. Then $q_{h}: X \times X \rightarrow[0, \infty)$ defined by $q_{h}:=\xi_{e} \circ h$ is a Q-function on $\left(X, d_{p}\right)$, where $d_{p}:=\xi_{e} \circ p$.
Proof. By $h(x, y) \in K, K \cap(-$ int $K)=\emptyset$, and (iii) of Lemma 6, we have $\xi_{e} \circ h(x, y) \geq 0$; that is, $q_{h}(x, y) \geq 0$, for all $x, y \in X$. Using (vii) and (ix) of Lemma 6 and (c2), we obtain

$$
\begin{array}{r}
\xi_{e} \circ h(x, z) \leq \xi_{e} \circ h(x, y)+\xi_{e} \circ h(y, z), \\
\forall x, y, z \in X .
\end{array}
$$

That is, $q_{h}$ satisfies (q1).

Next we prove that $q_{h}$ satisfies (q2). For this, let $x \in X$, $\left\{y_{n}\right\} \subset X$ such that $y_{n} \rightarrow y$ in $\left(X, d_{p}\right)$; that is, $d_{p}\left(y_{n}, y\right) \rightarrow 0$ as $n \rightarrow \infty$, and $q_{h}\left(x, y_{n}\right) \leq M$ for some $M=M(x)>0$. According to (i) of Theorem 8, it follows that $y_{n} \rightarrow y$ as $n \rightarrow$ $\infty$ in $(X, p)$. By $q_{h}\left(x, y_{n}\right) \leq M$ and (i) of Lemma 6 , we have $h\left(x, y_{n}\right) \preceq_{K} M e$. It follows from (c3) that $h(x, y) \preceq_{K} M e$. Again using (i) of Lemma 6, we obtain

$$
q_{h}(x, y)=\xi_{e} \circ h(x, y) \leq M .
$$

That is, $q_{h}$ satisfies (q2).

Finally, we show that $q_{h}$ satisfies (q3). Let $\varepsilon>0$ be given. Then $e \in$ int $K$ implies $\theta \ll \varepsilon e$. As $h$ satisfies the condition (c4), there is $\eta \in E$ with $\theta \ll \eta$ such that $h(z, x) \ll \eta$ and $h(z, y) \ll \eta$ imply $p(x, y) \ll \varepsilon e$. Since $\eta-\operatorname{int} K$ is a neighborhood of $\theta$, there exists $\delta>0$ such that $\delta e \in \eta-$ int $K$; that is, $\delta e \ll \eta$. Using (ii) of Lemma 6 , when $q_{h}(z, x)<\delta$ and $q_{h}(z, y)<\delta$, we have $h(z, x) \ll \delta e \ll \eta$ and $h(z, y) \ll \delta e \ll$ $\eta$, which imply $p(x, y) \ll \varepsilon e$. Again using (ii) of Lemma 6 , we obtain $d_{p}(x, y)=\xi_{e} \circ p(x, y)<\varepsilon$. Thus we have shown that $q_{h}$ satisfies (q3) and $q_{h}$ is a $Q$-function on $\left(X, d_{p}\right)$.

Similarly, we can deduce the following.

Theorem 10. Let $(X, p)$ be a tvs-cone metric space and let $h$ be a $w$-cone distance on $X$. Then $q_{h}: X \times X \rightarrow[0, \infty)$ defined by $q_{h}:=\xi_{e} \circ h$ is a $w$-distance on $\left(X, d_{p}\right)$, where $d_{p}:=\xi_{e} \circ p$.

\section{The Results for Maps on Metric Space}

Let $\Psi$ be the family of functions $\psi:[0,+\infty) \rightarrow[0,+\infty)$ satisfying the following conditions:

(i) $\psi$ is nondecreasing;

(ii) $\psi(0)=0$;

(iii) $\lim _{x \rightarrow+\infty}(x-\psi(x))=+\infty$;

(iv) $\lim _{t \rightarrow r^{+}} \psi(t)<r$ for all $r>0$.

It is obvious that if $\psi:[0,+\infty) \rightarrow[0,+\infty)$ is defined by $\psi(t)=\lambda t$ for some $\lambda \in[0,1)$, or $\psi(t)=\ln (t+1)$, then $\psi \in \Psi$.

Remark 11 (see [5, Remark 2.1]). If $\psi \in \Psi$, then we have that $\psi(r)<r$ and $\lim _{n \rightarrow+\infty} \psi^{n}(r)=0$ for all $r>0$.

Let $(X, d)$ be a metric space and $q$ be a $Q$-function on $X$. For $E \subset X$, we define $\delta_{q}(E)=\sup \{q(x, y): x, y \in E\}$.

Letting $f, g: X \rightarrow X$ be self-mappings, $f$ and $g$ are a $(\psi, q)$-quasi-contraction if there exists $\psi:[0,+\infty) \rightarrow$ $[0,+\infty)$ such that

$$
\begin{gathered}
q(f x, f y) \leq \max \{\psi(q(g x, g y)), \psi(q(g x, f x)), \\
\psi(q(g y, f y)), \psi(q(g x, f y)), \\
\psi(q(g y, f x))\}
\end{gathered}
$$

for all $x, y \in X$. 
Let us recall that the self-mappings $f$ and $g$ on $X$ are said to be weakly compatible if they commute at their coincidence point (i.e., $f g x=g f x$ whenever $f x=g x$ ). $f$ and $g$ are said to be compatible if

$$
\lim _{n \rightarrow+\infty} d\left(g f x_{n}, f g x_{n}\right)=0,
$$

whenever $\left\{x_{n}\right\}$ is a sequence in $x$ such that

$$
\lim _{n \rightarrow+\infty} g x_{n}=\lim _{n \rightarrow+\infty} f x_{n}=x .
$$

If $f$ and $g$ satisfy $f(X) \subset g(X)$ and $x_{0} \in X$, let us define $x_{1} \in X$ such that $f x_{0}=g x_{1}$. Having defined $x_{n} \in X$, let $x_{n+1} \in X$ be such that $f x_{n}=g x_{n+1}$. We say that $\left\{f x_{n}\right\}$ is a $f$-g-sequence of initial point $x_{0}$. Define

$$
\begin{gathered}
\mathcal{O}\left(x_{0}, n\right)=\left\{g x_{0}, f x_{0}, f x_{1}, \ldots, f x_{n}\right\}, \\
\mathcal{O}\left(x_{0}, \infty\right)=\left\{g x_{0}, f x_{0}, f x_{1}, \ldots\right\} .
\end{gathered}
$$

The following lemma is crucial in this paper.

Lemma 12. Let $(X, d)$ be a metric space and let $q$ be a $Q-$ function on $X$. Suppose that the self-mappings $f, g: X \rightarrow X$ are $a(\psi, q)$-quasi-contraction with $\psi \in \Psi$. Let $f(X) \subset g(X)$. For $x_{0} \in X$, let $\left\{f x_{n}\right\}$ be a $f$-g-sequence of initial point $x_{0}$. Then one has the following:

(i) for each $x_{0} \in X$ and $n \in \mathbf{N}$, there exist $k, l \in \mathbf{N}$ with $k, l \leq n$ such that

$$
\begin{aligned}
& \delta_{q}\left(\mathcal{O}\left(x_{0}, n\right)\right) \\
& \quad=\max \left\{q\left(g x_{0}, g x_{0}\right), q\left(g x_{0}, f x_{k}\right), q\left(f x_{l}, g x_{0}\right)\right\} ;
\end{aligned}
$$

(ii) for each $x_{0} \in X$, there exists $c>0$ such that

$$
\delta_{q}\left(\mathcal{O}\left(x_{0}, \infty\right)\right) \leq c
$$

(iii) for each $x_{0} \in X,\left\{f x_{n}\right\}$ is a Cauchy sequence. If $\left\{f x_{n}\right\}$ converges to some $y \in X$, then

$$
q\left(f x_{n}, y\right) \leq \psi^{n}(c),
$$

for all $n \geq 0$, where $\psi^{0}(c)=c$.

Proof. (i) Let $x_{0} \in X$ and $n \in \mathbf{N}$. Since $f$ and $g$ are a $(\psi, q)$ quasi-contraction with $\psi \in \Psi$, for every $0 \leq i, j \leq n$, we have

$$
\begin{aligned}
& q\left(f x_{i}, f x_{j}\right) \\
& \leq \max \left\{\psi\left(q\left(g x_{i}, g x_{j}\right)\right), \psi\left(q\left(g x_{i}, f x_{i}\right)\right),\right. \\
& \psi\left(q\left(g x_{j}, f x_{j}\right)\right), \psi\left(q\left(g x_{i}, f x_{j}\right)\right), \\
& \left.\psi\left(q\left(g x_{j}, f x_{i}\right)\right)\right\} \\
& \leq \psi\left(\delta_{q}\left(\mathcal{O}\left(x_{0}, n\right)\right)\right) \\
& <\delta_{q}\left(\mathcal{O}\left(x_{0}, n\right)\right) .
\end{aligned}
$$

This implies that

$$
\begin{aligned}
\delta_{q} & \left(\mathcal{O}\left(x_{0}, n\right)\right) \\
& =\max \left\{q\left(g x_{0}, g x_{0}\right), q\left(g x_{0}, f x_{k}\right), q\left(f x_{l}, g x_{0}\right)\right\},
\end{aligned}
$$

for some $0 \leq k, l \leq n$.

(ii) By property (iii) of the function $\psi$, for $h=$ $\max \left\{q\left(g x_{0}, g x_{0}\right), q\left(g x_{0}, f x_{0}\right), q\left(f x_{0}, g x_{0}\right)\right\}$, there is a $c>h$ such that $t-\psi(t)>h$, for all $t>c$. Therefore, for each $n \geq 1$,

$$
\begin{aligned}
& \delta_{q}\left(\mathcal{O}\left(x_{0}, n\right)\right) \\
&=\max \left\{q\left(g x_{0}, g x_{0}\right), q\left(g x_{0}, f x_{k}\right), q\left(f x_{l}, g x_{0}\right)\right\} \\
& \leq \max \left\{q\left(g x_{0}, g x_{0}\right), q\left(g x_{0}, f x_{0}\right)\right. \\
&\left.+q\left(f x_{0}, f x_{k}\right), q\left(f x_{l}, f x_{0}\right)+q\left(f x_{0}, g x_{0}\right)\right\} \\
& \leq h+\max \left\{q\left(f x_{0}, f x_{k}\right), q\left(f x_{l}, f x_{0}\right)\right\} \\
& \leq h+\psi\left(\delta_{q}\left(\mathcal{O}\left(x_{0}, n\right)\right)\right) .
\end{aligned}
$$

Thus,

$$
\delta_{q}\left(\mathcal{O}\left(x_{0}, n\right)\right)-\psi\left(\delta_{q}\left(\mathcal{O}\left(x_{0}, n\right)\right)\right) \leq h
$$

for all $n \in \mathbf{N}$. It follows that

$$
\delta_{q}\left(\mathcal{O}\left(x_{0}, n\right)\right) \leq c, \quad \delta_{q}\left(\mathcal{O}\left(x_{0}, \infty\right)\right) \leq c .
$$

(iii) Define $\mathcal{O}\left(f x_{k}\right)=\left\{f x_{k}, f x_{k+1}, \ldots\right\}$ for every $k \geq 0$. Obviously $\delta_{q}\left(\mathcal{O}\left(f x_{k}\right)\right) \leq \psi\left(\delta_{q}\left(\mathcal{O}\left(f x_{k-1}\right)\right)\right)$ for all $k \geq 1$. Consequently, for all $m, n \in \mathbf{N}$ with $m>n$,

$$
\begin{aligned}
q\left(f x_{n}, f x_{m}\right) & \leq \delta_{q}\left(\mathcal{O}\left(f x_{n}\right)\right) \leq \psi\left(\delta_{q}\left(\mathcal{O}\left(f x_{n-1}\right)\right)\right) \\
& \leq \cdots \leq \psi^{n}\left(\delta_{q}\left(\mathcal{O}\left(f x_{0}\right)\right)\right) \\
& \leq \psi^{n}\left(\delta_{q}\left(\mathcal{O}\left(x_{0}, \infty\right)\right)\right) \\
& \leq \psi^{n}(c) .
\end{aligned}
$$

From Lemma 2(iii) and Remark 11, it follows that $\left\{f x_{n}\right\}$ is a Cauchy sequence. If it converges to $y \in X$, then (19) implies that $q\left(f x_{n-1}, y\right) \leq \psi^{n-1}(c)$.

Theorem 13. Let $(X, d)$ be a complete metric space and let $q$ be a $Q$-function on X. Suppose that the self-mappings $f, g$ : $X \rightarrow X$ are $a(\psi, q)$-quasi-contraction with $\psi \in \Psi$. Let $f(X) \subset g(X)$, and let

(D1) for every $y \in X$ with $f y \neq g y$

$$
\inf \{q(g x, y)+q(g x, f x): x \in X\}>0 .
$$

If $f$ and $g$ are weakly compatible, then the mappings $f$ and $g$ have a unique common fixed point $u$ in $X$ and $q(u, u)=0$.

Proof. Let $x_{0} \in X$ be fixed. As $f(X) \subset g(X)$, we construct a $f$-g-sequence $\left\{f x_{n}\right\}$ of initial point $x_{0}$. Using Lemma 12(iii), 
we see that $\left\{f x_{n}\right\}$ is a Cauchy sequence. Since $X$ is complete, there exists $y \in X$ such that $f x_{n} \rightarrow y$ and also $g x_{n} \rightarrow y$.

Let us prove that $f y=g y$. If $f y \neq g y$, then (D1), (13), and (19) imply

$$
\begin{aligned}
0 & <\inf \{q(g x, y)+q(g x, f x): x \in X\} \\
& \leq \inf \left\{q\left(g x_{n}, y\right)+q\left(g x_{n}, f x_{n}\right): n \in \mathbf{N}\right\} \\
& =\inf \left\{q\left(f x_{n-1}, y\right)+q\left(f x_{n-1}, f x_{n}\right): n \in \mathbf{N}\right\} \\
& \leq \inf \left\{\psi^{n-1}(c)+\psi^{n-1}(c): n \in \mathbf{N}\right\} \\
& =0 .
\end{aligned}
$$

This is a contradiction. Hence $f y=g y$.

Thus

$$
q(g y, g y)=q(f y, f y) \leq \psi(q(g y, g y))
$$

and so $q(g y, g y)=0$. Similarly, $q\left(g^{2} y, g^{2} y\right)=0$.

Now, we show that $u=g y$ is a common fixed point for $f$ and $g$. Since $f$ and $g$ are weakly compatible, we deduce that

$$
f u=f g y=g f y=g^{2} y=g u .
$$

From this, we obtain that

$$
\begin{aligned}
q(f u, f y) & \leq \max \{\psi(q(f u, f y)), \psi(q(f y, f u))\} \\
& =\psi \max \{q(f u, f y), q(f y, f u)\} \\
q(f y, f u) & \leq \psi(\max \{q(f u, f y), q(f y, f u)\}) .
\end{aligned}
$$

From (24) and (25), it follows that $q(f u, f y)=0$ and $q(f y, f u)=0$. Using $q(f u, u)=q(f u, f y)=0, q(f u, f u)=$ $q\left(g^{2} y, g^{2} y\right)=0$, and Lemma 2(i), we obtain that $f u=u$. From (23), it follows that $f u=g u=u$.

To prove the uniqueness of the common fixed point of $f$ and $g$, let us suppose that there exists $v \in X$ and $f v=g v=v$. From the definition of $(\psi, q)$-quasi-contraction, it follows that

$$
\begin{gathered}
q(u, u)=q(f u, f u) \leq \psi(q(u, u)), \\
q(u, v)=q(f u, f v) \leq \psi(\max \{q(u, v), q(v, u)\}), \\
q(v, u)=q(f v, f u) \leq \psi(\max \{q(u, v), q(v, u)\}) .
\end{gathered}
$$

Thus $q(u, u)=q(u, v)=q(v, u)=0$. By Lemma 2(i), we conclude that $u=v$ and $q(u, u)=0$.

Theorem 14. Let $(X, d)$ be a metric space and let $q$ be a $Q$ function on $X$. Suppose that the self-mappings $f, g: X \rightarrow X$ are $a(\psi, q)$-quasi-contraction with $\psi \in \Psi$. Let $f(X) \subset g(X)$, and let

(D2) for every $z \in X$ with $f z \neq g z$

$$
\inf \{q(f x, g z)+q(g x, f x): x \in X\}>0
$$

If $f(X)$ or $g(X)$ is a complete subspace of $X$ and $f$ and $g$ are weakly compatible, then the mappings $f$ and $g$ have a unique common fixed point $u$ in $X$ and $q(u, u)=0$.
Proof. Let $x_{0} \in X$ be fixed. As $f(X) \subset g(X)$, we construct a $f$-g-sequence $\left\{f x_{n}\right\}$ of initial point $x_{0}$. Using Lemma 12 (iii), we see that $\left\{f x_{n}\right\}$ is a Cauchy sequence. Since $f(X)$ or $g(X)$ is a complete subspace of $X$, there exists $y \in g(X)$ such that $f x_{n} \rightarrow y$ and also $g x_{n} \rightarrow y$. Let $z \in X$ be such that $g z=y$.

Let us prove that $f z=g z$. If $f z \neq g z$, then (D2), (13), and (19) imply

$$
\begin{aligned}
0 & <\inf \{q(f x, g z)+q(g x, f x): x \in X\} \\
& \leq \inf \left\{q\left(f x_{n}, g z\right)+q\left(g x_{n}, f x_{n}\right): n \in \mathbf{N}\right\} \\
& =\inf \left\{q\left(f x_{n}, y\right)+q\left(f x_{n-1}, f x_{n}\right): n \in \mathbf{N}\right\} \\
& \leq \inf \left\{\psi^{n}(c)+\psi^{n-1}(c): n \in \mathbf{N}\right\} \\
& =0 .
\end{aligned}
$$

This is a contradiction. Hence $f z=g z$.

Similar to the proof of Theorem 13, we can conclude that $u=f z$ is a unique common fixed point and $q(u, u)=0$.

Theorem 15. Let $(X, d)$ be a complete metric space and let $q$ be a $Q$-function on $X$. Suppose that the self-mappings $f, g$ : $X \rightarrow X$ are $a(\psi, q)$-quasi-contraction with $\psi \in \Psi$. Let $f(X) \subset g(X)$ and let $f$ and $g$ be continuous mappings. If $f$ and $g$ are compatible, then the mappings $f$ and $g$ have a unique common fixed point $u$ in $X$ and $q(u, u)=0$.

Proof. Let $x_{0} \in X$ be fixed. As $f(X) \subset g(X)$, we construct a $f$-g-sequence $\left\{f x_{n}\right\}$ of initial point $x_{0}$. Using Lemma 12 (iii), we see that $\left\{f x_{n}\right\}$ is a Cauchy sequence. Since $X$ is complete, there exists $y \in X$ such that $f x_{n} \rightarrow y$ and also $g x_{n} \rightarrow y$. Since $f$ and $g$ are compatible, we have

$$
\lim _{n \rightarrow \infty} d\left(g f x_{n}, f g x_{n}\right)=0
$$

Using the continuousness of $f$ and $g$, we deduce that $g f x_{n}=$ $g y$ and $f g x_{n}=f y$. From (29), it follows that $g y=f y$.

Similar to the proof of Theorem 13, we can conclude that $u=f y$ is a unique common fixed point and $q(u, u)=0$.

Remark 16. From the proof of Theorems 13 and 15, we see that the completeness of the space $X$ can be replaced by the completeness of the subspace $f(X)$ or $g(X)$.

If we take $\psi(t)=k t$ for some $k \in[0,1)$ in Theorems $13-15$, then we have the following results.

Corollary 17. Let $X$ be a complete metric space with metric $d$ and let $q$ be a Q-function on $X$. Let $f$ and $g$ be the mappings of $X$ into itself satisfying $f(X) \subset g(X)$ and

$$
\begin{aligned}
q(f x, f y) \leq k \max & \{q(g x, g y), q(g x, f x), \\
& q(g y, f y), \\
& q(g x, f y), q(g y, f x)\}
\end{aligned}
$$

for all $x, y \in X$ and some $k \in[0,1)$.

Assume that either of the following holds:

(i) $f$ and $g$ are weakly compatible and satisfy the condition (D1);

(ii) $f$ and $g$ are compatible and continuous. 
Then the mappings $f$ and $g$ have a unique common fixed point $u$ in $X$ and $q(u, u)=0$.

Corollary 18. Let $X$ be a metric space with metric $d$ and let $q$ be a $Q$-function on $X$. Let $f$ and $g$ be the mappings of $X$ into itself satisfying $f(X) \subset g(X)$ and

$$
\begin{gathered}
q(f x, f y) \\
\leq k \max \{q(g x, g y), q(g x, f x), q(g y, f y), \\
q(g x, f y), q(g y, f x)\}
\end{gathered}
$$

for all $x, y \in X$ and some $k \in[0,1)$.

Assume that either of the following holds:

(i) $f$ and $g$ are weakly compatible and satisfy the condition (D1);

(ii) $f$ and $g$ are weakly compatible and satisfy the condition (D2);

(iii) $f$ and $g$ are compatible and continuous.

If $f(X)$ or $g(X)$ is complete, then the mappings $f$ and $g$ have a unique common fixed point $u$ in $X$ and $q(u, u)=0$.

In the results presented by di Bari and Vetro [5], the condition (D1) or (D2) is not required. In fact, the conditions (D1) and (D2) always hold when $q=d$.

Proposition 19. Let $(X, d)$ be a metric space and let the selfmappings $f, g: X \rightarrow X$ be such that $f(X) \subset g(X)$. If $f$ and $g$ are $a(\psi, d)$-quasi-contraction with $\psi \in \Psi$, then for every $z \in X$ with $f z \neq g z$

$$
\inf \{d(f x, g z)+d(g x, f x): x \in X\}>0 .
$$

Proof. Suppose that there exists $z \in X$ with $f z \neq g z$ and

$$
\inf \{d(f x, g z)+d(g x, f x): x \in X\}=0 .
$$

Then there exists a sequence $\left\{x_{n}\right\}$ in $X$ such that

$$
\lim _{n \rightarrow \infty}\left[d\left(f x_{n}, g z\right)+d\left(g x_{n}, f x_{n}\right)\right]=0 .
$$

It follows that $f x_{n} \rightarrow g z$ and $d\left(g x_{n}, f x_{n}\right) \rightarrow 0$, and so $g x_{n} \rightarrow g z$. Define $d=d(f z, g z)>0$.

Thus, for $n$ sufficiently large, we have

$$
\max \left\{d\left(g x_{n}, g z\right), d\left(g x_{n}, f x_{n}\right), d\left(g z, f x_{n}\right)\right\}<d .
$$

From

$$
\begin{aligned}
& d\left(f x_{n}, f z\right) \\
& \leq \max \left\{\psi\left(d\left(g x_{n}, g z\right)\right), \psi\left(d\left(g x_{n}, f x_{n}\right)\right),\right. \\
& \psi(d(g z, f z)), \psi\left(d\left(g x_{n}, f z\right)\right), \\
& \left.\psi\left(d\left(g z, f x_{n}\right)\right)\right\},
\end{aligned}
$$

we deduce that

$$
d\left(f x_{n}, f z\right) \leq \psi\left(\max \left\{d, d\left(g x_{n}, f z\right)\right\}\right) .
$$

Since $d\left(g x_{n}, f z\right) \rightarrow d(g z, f z)=d$, we get $\max \{d$, $\left.d\left(g x_{n}, f z\right)\right\} \rightarrow d^{+}$. Using the property (iv) of the function $\psi$, we see that

$$
\begin{aligned}
d & =d(g z, f z)=\lim _{n \rightarrow \infty} d\left(f x_{n}, f z\right) \\
& \leq \lim _{n \rightarrow \infty} \psi\left(\max \left\{d, d\left(g x_{n}, f z\right)\right\}\right)<d,
\end{aligned}
$$

which is a contradiction.

Proposition 20. Let $(X, d)$ be a metric space and let the selfmappings $f, g: X \rightarrow X$ be such that $f(X) \subset g(X)$. Suppose that $f$ and $g$ are a weakly compatible pair and $a(\psi, d)$-quasicontraction with $\psi \in \Psi$. If $f(X)$ or $g(X)$ is closed subspace of $X$, then for every $y \in X$ with $f y \neq g y$

$$
\inf \{d(g x, y)+d(g x, f x): x \in X\}>0 .
$$

Proof. Suppose that there exists $y \in X$ with $f y \neq g y$ and

$$
\inf \{d(g x, y)+d(g x, f x): x \in X\}=0 .
$$

Then there exists a sequence $\left\{x_{n}\right\}$ in $X$ such that

$$
\lim _{n \rightarrow \infty}\left[d\left(g x_{n}, y\right)+d\left(g x_{n}, f x_{n}\right)\right]=0
$$

It follows that $g x_{n} \rightarrow y$ and $d\left(g x_{n}, f x_{n}\right) \rightarrow 0$, and so $f x_{n} \rightarrow y$. Since $f(X) \subset g(X)$, and $f(X)$ or $g(X)$ is closed, we have $y \in g(X)$. Thus there exists $z \in X$ such that $y=g z$.

Let us prove that $f z=g z$. If $f z \neq g z$, then $d=$ $d(g z, f z)>0$. Similar to the proof of Proposition 19, we can get a contradiction. Thus $f z=g z=y$.

Since $f$ and $g$ are weakly compatible, we obtain

$$
g y=g f z=f g z=f y,
$$

which is a contradiction with $g y \neq f y$.

Remark 21. Using Theorem 13 and Proposition 20, or Theorem 14 and Proposition 19, we get Theorem 2.2 of di Bari and Vetro [5].

Remark 22. In Corollary 17 we use the assumption that $f$ and $g$ are weakly compatible, which is weaker than the assumption that $f$ and $g$ commutes in Theorem 3.1 of Ilic and Rakocevic [4].

\section{The Results for Maps on Tvs-Cone Metric Space}

We denote with $\Psi_{K}$ the set of all functions $\phi: K \rightarrow K$ which have the following properties:

(i) $\phi$ is nondecreasing, that is, $\phi(x) \preceq_{K} \phi(y)$ whenever $x, y \in K$ with $x \preceq_{K} y$;

(ii) $\phi(\theta)=\theta$;

(iii) $\lim _{t \rightarrow+\infty}\left(t-\xi_{e}(\phi(t e))\right)=+\infty$;

(iv) $\lim _{t \rightarrow r^{+}} \phi(t e) \ll r e$ for all $r>0$.

We denote with $\Phi_{K}$ the set of all functions $\varphi: K \rightarrow K$ which have the following properties (see [5]): 
(i) $\varphi(\theta)=\theta$;

(ii) $\varphi(t) \ll t$ for all $t \in$ int $K$;

(iii) $\lim _{t \rightarrow+\infty}\left(t-\xi_{e}(\varphi(t e))\right)=+\infty$ for some $e \in \operatorname{int} K$;

(iv) if $x_{n} \rightarrow x, x_{n}, x \in$ int $K$, then there exists $\lambda(x) \in$ $(0,1)$ and $n_{0} \in \mathbf{N}$ such that $\varphi\left(x_{n}\right) \preceq_{K} \lambda(x)$ for all $n \geq$ $n_{0}$.

Remark 23 (see [5, Remark 2.3 and Theorem 3.5]). If $\varphi \in \Phi_{K}$, then the function $\phi: K \rightarrow K$ is defined by

$$
\phi(x)=\phi\left(\xi_{e}(x) e\right)=\sup _{t \in\left[0, \xi_{e}(x)\right]} \varphi(t e) \in \Psi_{K} .
$$

Let $(X, p)$ be a tvs-cone metric space and let $h$ be a $c$ distance on $X$. Let $f, g: X \rightarrow X$ be self-mappings. Then $f$ and $g$ are called a $(\phi, h)$-quasi-contraction if there exists $\phi \in \Psi_{K}$ such that for all $x, y \in X$

$$
h(f x, f y) \preceq_{K} u,
$$

where

$$
\begin{aligned}
u \in\{\phi(h(g x, g y)), \phi(h(g x, f x)), \\
\phi(h(g y, f y)), \\
\phi(h(g x, f y)), \phi(h(g y, f x))\} .
\end{aligned}
$$

Now we give several Lemmas as follows.

Lemma 24. Let $(X, p)$ be a tvs-cone metric space and let $h$ be a c-distance on $X$. Suppose that the mappings $f, g: X \rightarrow X$ satisfy the following condition:

(C1) if $f y \neq g y$, there exists $c \in$ int $K$ such that

$$
c \preceq_{K} h(g x, y)+h(g x, f x) \quad \forall x \in X .
$$

Then for every $y \in X$ with $f y \neq g y$

$$
\inf \left\{q_{h}(g x, y)+q_{h}(g x, f x): x \in X\right\}>0,
$$

where $q_{h}:=\xi_{e} \circ h$.

Proof. Take $y \in X$ with $f y \neq g y$. Using (vii) and (ix) of Lemma 6 and the assumption (C1), we have

$$
\begin{aligned}
\xi_{e}(c) & \leq \xi_{e} \circ(h(g x, y)+h(g x, f x)) \\
& \leq \xi_{e} \circ h(g x, y)+\xi_{e} \circ h(g x, f x) \\
& =q_{h}(g x, y)+q_{h}(g x, f x),
\end{aligned}
$$

for all $x \in X$. Using (iv) of Lemma $6, c \in \operatorname{int} K$ and int $K \cap$ $(-K)=\emptyset$ imply that

$$
\begin{gathered}
\inf \left\{q_{h}(g x, y)+q_{h}(g x, f x): x \in X\right\} \\
\geq \xi_{e}(c)>0 .
\end{gathered}
$$

Similarly, we can deduce the following.
Lemma 25. Let $(X, p)$ be a tvs-cone metric space and let $h$ be a c-distance on $X$. Suppose that the mappings $f, g: X \rightarrow X$ satisfy the following condition:

(C2) if $f z \neq g z$, there exists $c \in$ int $K$ such that

$$
c \preceq_{K} h(f x, g z)+h(g x, f x) \quad \forall x \in X .
$$

Then for every $z \in X$ with $f z \neq g z$

$$
\inf \left\{q_{h}(f x, g z)+q_{h}(g x, f x): x \in X\right\}>0,
$$

where $q_{h}:=\xi_{e} \circ h$.

Lemma 26. Let $(X, p)$ be a tvs-cone metric space and let $f, g$ : $X \rightarrow X$ be self-mappings. Then

(i) $f$ is continuous in $(X, p)$ if and only if so is in $\left(X, d_{p}\right)$;

(ii) $f$ and $g$ are compatible in $(X, p)$ if and only if so is in $\left(X, d_{p}\right)$.

Proof. From (i) of Theorem 8, the conclusions are obvious.

Lemma 27. Let $(X, p)$ be a tvs-cone metric space and let $h$ be a c-distance on $X$. Suppose the self-mappings $f, g: X \rightarrow X$ are $a(\phi, h)$-quasi-contraction with $\phi \in \Psi_{K}$. Then there exists $\psi \in \Psi$ such that $f$ and $g$ are $\left(\psi, q_{h}\right)$-quasi-contraction, where $q_{h}:=\xi_{e} \circ h$.

Proof. We choose $e \in$ int $K$ such that $\lim _{t \rightarrow+\infty}\left[t-\xi_{e}\right.$ $(\phi(t e))]=+\infty$. Define $\psi:[0,+\infty) \rightarrow[0,+\infty)$ as $\psi(t)=$ $\xi_{e}(\phi(t e))$. From Lemma 6 and the properties of the function $\phi$, the function $\psi$ has the following properties:

(i) $\psi$ is nondecreasing;

(ii) $\psi(0)=\xi_{e}(\phi(\theta))=\xi_{e}(\theta)=0$;

(iii) $\lim _{t \rightarrow+\infty}(t-\psi(t))=\lim _{t \rightarrow+\infty}\left(t-\xi_{e}(\phi(t e))\right)=+\infty$;

(iv) $\lim _{t \rightarrow r^{+}} \psi(t)=\lim _{t \rightarrow r^{+}} \xi_{e}(\phi(t e))=\xi_{e}\left(\lim _{t \rightarrow r^{+}}\right.$ $\phi(t e))<\xi_{e}(r e)=r$ for all $r>0$.

Then $\psi \in \Psi$. Notice that $h(x, y) \preceq_{K} \xi_{e}(h(x, y)) e=q_{h}$ $(x, y) e$, so

$$
\xi_{e}(\phi(h(x, y))) \leq \xi_{e}\left(\phi\left(q_{h}(x, y) e\right)\right)=\psi\left(q_{h}(x, y)\right),
$$

for all $x, y \in X$. Thus, from $h(f x, f y) \preceq_{K} u$ where $u$ satisfies (45), we deduce that

$$
\begin{gathered}
q_{h}(f x, f y)=\xi_{e}(h(f x, f y)) \leq \xi_{e}(u) \\
\leq \max \left\{\psi\left(q_{h}(g x, g y)\right), \psi\left(q_{h}(g x, f x)\right),\right. \\
\psi\left(q_{h}(g y, f y)\right), \psi\left(q_{h}(g x, f y)\right), \\
\left.\psi\left(q_{h}(g y, f x)\right)\right\} .
\end{gathered}
$$

That is, $f$ and $g$ are a $\left(\psi, q_{h}\right)$-quasi-contraction. 
Using Lemmas 24, 26, and 27 and Theorems 13 and 15, we obtain the following.

Theorem 28. Let $(X, p)$ be a complete tvs-cone metric space and let $h$ be a $c$-distance on $X$. Let $f$ and $g$ be the mappings of $X$ into itself satisfying $f(X) \subset g(X)$ and $a(\phi, h)$-quasicontraction with $\phi \in \Psi_{K}$.

Assume that either of the following holds:

(i) $f$ and $g$ are weakly compatible and satisfy the condition (C1);

(ii) $f$ and $g$ are compatible and continuous.

Then the mappings $f$ and $g$ have a unique common fixed point $u$ in $X$ and $h(u, u)=\theta$.

Using Lemmas 24-27, Theorems 13-15, and Remark 16, we have the following.

Theorem 29. Let $(X, p)$ be a tvs-cone metric space and let $h$ be a $c$-distance on $X$. Let $f$ and $g$ be the mappings of $X$ into itself satisfying $f(X) \subset g(X)$ and $a(\phi, h)$-quasi-contraction with $\phi \in \Psi_{K}$.

Assume that either of the following holds:

(i) $f$ and $g$ are weakly compatible and satisfy the condition (C1);

(ii) $f$ and $g$ are weakly compatible and satisfy the condition (C2);

(iii) $f$ and $g$ are compatible and continuous.

If $f(X)$ or $g(X)$ is complete, then the mappings $f$ and $g$ have a unique common fixed point $u$ in $X$ and $h(u, u)=\theta$.

Theorem 30. Let $(X, p)$ be a complete tvs-cone metric space and let $h$ be a c-distance on $X$. Let $f$ and $g$ be the mappings of $X$ into itself satisfying $f(X) \subset g(X)$ and the following condition:

(a) $h(f x, f y) \preceq_{K} A h(g x, g y)+B h(g x, f x)+C h(g y, f y)+$ $\operatorname{Dh}(g x, f y)+E h(g y, f x)$

for all $x, y \in X$, where $A, B, C, D, E$ are nonnegative constants such that $A+B+C+D+E<1$.

Assume that either of the following holds:

(i) if $y \neq f y$, there exists $c \in$ int $K$ such that

$$
c \preceq_{K} h(g x, y)+h(g x, f x) \quad \forall x \in X ;
$$

(ii) $f$ and $g$ are compatible and continuous.

Then the mappings $f$ and $g$ have a unique common fixed point $u$ in $X$ and $h(u, u)=\theta$.
Proof. Put $d_{p}:=\xi_{e} \circ p$ and $q_{h}:=\xi_{e} \circ h$. Using Lemma 6 and the assumption (a), we obtain

$$
\begin{gathered}
q_{h}(f x, f y) \\
\leq A q_{h}(g x, g y)+B q_{h}(g x, f x)+C q_{h}(g y, f y) \\
+D q_{h}(g x, f y)+E q_{h}(g y, f x) \\
\leq k \max \left\{q_{h}(g x, g y), q_{h}(g x, f x), q_{h}(g y, f y),\right. \\
\left.q_{h}(g x, f y), q_{h}(g y, f x)\right\}
\end{gathered}
$$

for all $x, y \in X$, where $k=A+B+C+D+E \in[0,1)$. By Theorem 7 , Theorem 8 , and Theorem 9 , we see that $\left(X, d_{p}\right)$ is a complete metric space and $q_{h}$ is a $Q$-function on $\left(X, d_{p}\right)$. Applying Corollary 17 in $\left(X, d_{p}\right)$, we deduce that $f$ and $g$ have a unique common fixed point $u$ in $X$. It is easy to see that $h(u, u)=\theta$ from the condition (a).

If we take $g=I_{X}$, the identity mapping on $X$, and $f=T$, then we get the following corollaries.

Corollary 31. Let $(X, p)$ be a complete tvs-cone metric space and let $h$ be a $c$-distance on $X$. Suppose that the mapping $T$ : $X \rightarrow X$ satisfies that, for some constant $k \in[0,1)$ and for every $x, y \in X$, there exists

$$
u \in\{h(x, z), h(x, T x), h(y, T y), h(x, T y), h(y, T x)\}
$$

such that

$$
h(T x, T y) \preceq_{K} k u .
$$

Assume that either of the following holds:

(i) if $y \neq T y$, there exists $c \in$ int $K$ such that

$$
c \preceq_{K} h(x, y)+h(x, T x) \quad \forall x \in X ;
$$

(ii) $T$ is continuous.

Then $T$ has a unique fixed point $u$ in $X$ and $h(u, u)=\theta$.

Corollary 32. Let $(X, p)$ be a tvs-cone metric space and let $h$ be a c-distance on $X$. Suppose that the mapping $T: X \rightarrow X$ satisfies that, for some constant $k \in[0,1)$ and for every $x, y \in$ $X$, there exists

$$
u \in\{h(x, z), h(x, T x), h(y, T y), h(x, T y), h(y, T x)\}
$$

such that

$$
h(T x, T y) \preceq_{K} k u .
$$

Assume that either of the following holds:

(i) if $y \neq T y$, there exists $c \in$ int $K$ such that

$$
c \preceq_{K} h(x, y)+h(x, T x) \quad \forall x \in X ;
$$


(ii) if $z \neq T z$, there exists $c \in$ int $K$ such that

$$
c \preceq_{K} h(T x, y)+h(x, T x) \quad \forall x \in X ;
$$

(iii) $T$ is continuous.

If $f(X)$ or $g(X)$ is complete, then $T$ has a unique fixed point $u$ in $X$ and $h(u, u)=\theta$.

Corollary 33. Let $(X, p)$ be a complete tvs-cone metric space and let $h$ be a c-distance on $X$. Suppose that a mapping $T$ : $X \rightarrow X$ satisfies the following condition:

(a) $h(x, y) \preceq_{K} A h(x, y)+B h(x, T x)+C h(y, T y)+$ $\operatorname{Dh}(x, T y)+E h(y, T x)$

for all $x, y \in X$, where $A, B, C, D, E$ are nonnegative constants such that $A+B+C+D+E<1$.

Assume that either of the following holds:

(i) if $y \neq T y$, there exists $c \in$ int $K$ such that

$$
c \preceq_{K} h(x, y)+h(x, T x) \quad \forall x \in X ;
$$

(ii) $T$ is continuous.

Then $T$ has a unique fixed point in $X$. If T $v=v$, then $h(v, v)=$ $\theta$.

Remark 34. As can be seen, the results of Corollary 31 and Corollary 32 provide an answer to Question 1.

Remark 35. Using Corollary 33, we can obtain [15, Theorem 2] and [16, Theorem 3.3]. Moreover, we see that the assumption $A+B+C+2 D+2 E<1$ is weakened to $A+B+C+D+E<$ 1 and the assumption $h(T y, T x) \preceq_{K} A h(y, x)+B h(T x, x)+$ $C h(T y, x)+D h(T y, y)+E h(T x, y)$ is removed in $[15$, Theorem 2].

\section{Conflict of Interests}

The author declares that there is no conflict of interests regarding the publication of this paper.

\section{Acknowledgments}

The author is grateful to the anonymous referees for useful comments and suggestions, which greatly improved the paper. This work was supported in part by Scientific Studies of Higher Education Institution of Inner Mongolia (NJZZ13019) and in part by Program of Higher-Level Talents of Inner Mongolia University (30105-125150 and 30105-135117).

\section{References}

[1] O. Kada, T. Suzuki, and W. Takahashi, "Nonconvex minimization theorems and fixed point theorems in complete metric spaces," Mathematica Japonica, vol. 44, no. 2, pp. 381-391, 1996.

[2] S. Al-Homidan, Q. H. Ansari, and J. Yao, "Some generalizations of Ekeland-type variational principle with applications to equilibrium problems and fixed point theory," Nonlinear Analysis: Theory, Methods and Applications, vol. 69, no. 1, pp. 126-139, 2008.
[3] J. Ume, "Fixed point theorems related to Ćirićs contraction principle," Journal of Mathematical Analysis and Applications, vol. 225, no. 2, pp. 630-640, 1998.

[4] D. Ilic and V. Rakocevic, "Common fixed points for maps on metric space with $w$-distance," Applied Mathematics and Computation, vol. 199, no. 2, pp. 599-610, 2008.

[5] C. di Bari and P. Vetro, "Nonlinear quasi-contractions of Ciric type," Fixed Point Theory, vol. 13, no. 2, pp. 453-459, 2012.

[6] W. S. Du, "A note on cone metric fixed point theory and its equivalence," Nonlinear Analysis: Theory, Methods \& Applications, vol. 72, no. 5, pp. 2259-2261, 2010.

[7] P. P. Zabrejko, "K-metric and K-normed spaces: survey," Universitat de Barcelona: Collectanea Mathematica, vol. 48, no. 4-6, pp. 825-859, 1997.

[8] L. Huang and X. Zhang, "Cone metric spaces and fixed point theorems of contractive mappings," Journal of Mathematical Analysis and Applications, vol. 332, no. 2, pp. 1468-1476, 2007.

[9] S. Janković, Z. Kadelburg, and S. Radenović, "On cone metric spaces: a survey," Nonlinear Analysis: Theory, Methods \& Applications, vol. 74, no. 7, pp. 2591-2601, 2011.

[10] A. Amini-Harandi and M. Fakhar, "Fixed point theory in cone metric spaces obtained via the scalarization method," Computers \& Mathematics with Applications, vol. 59, no. 11, pp. 3529-3534, 2010.

[11] Z. Kadelburg, S. Radenovic, and V. Rakočević, "A note on the equivalence of some metric and cone metric fixed point results," Applied Mathematics Letters, vol. 24, no. 3, pp. 370-374, 2011.

[12] Y. Q. Feng and W. Mao, "The equivalence of cone metric spaces and metric spaces," Fixed Point Theory, vol. 11, no. 2, pp. 259264, 2010.

[13] Y. J. Cho, R. Saadati, and S. H. Wang, "Common fixed point theorems on generalized distance in ordered cone metric spaces," Computers and Mathematics with Applications, vol. 61, no. 4, pp. 1254-1260, 2011.

[14] L. Ćirić, H. Lakzian, and V. Rakočević, "Fixed point theorems for $w$-cone distance contraction mappings in tvs-cone metric spaces," Fixed Point Theory and Applications, vol. 2012, article 3, 2012.

[15] M. Đorđević, D. Đorić, Z. Kadelburg, S. Radenović, and D. Spasić, "Fixed point results under c-distance in tvs-cone metric spaces," Fixed Point Theory and Applications, vol. 2011, article 29, 2011.

[16] Z. M. Fadail, A. G. B. Ahmad, and Z. Golubovi, "Fixed point theorems of single-valued mapping for $c$-distance in cone metric spaces," Abstract and Applied Analysis, vol. 2012, Article ID 826815, 11 pages, 2012.

[17] A. Göpfert, H. Riahi, C. Tammer, and C. Zalinescu, Variational Methods in Partially Ordered Spaces, Springer, New York, NY, USA, 2003.

[18] G. Y. Chen, X. X. Huang, and X. Q. Yang, Vector Optimization, Springer, Berlin, Germany, 2005.

[19] W. S. Du, "On some nonlinear problems induced by an abstract maximal element principle," Journal of Mathematical Analysis and Applications, vol. 347, no. 2, pp. 391-399, 2008.

[20] A. Göpfert, C. Tammer, and C. Zălinescu, "On the vectorial Ekeland's variational principle and minimal points in product spaces," Nonlinear Analysis: Theory, Methods \& Applications, vol. 39, no. 7, pp. 909-922, 2000. 


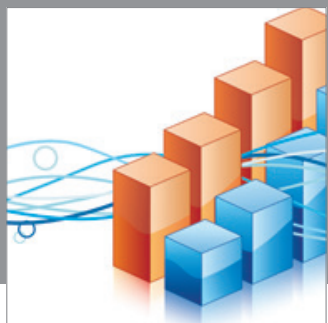

Advances in

Operations Research

mansans

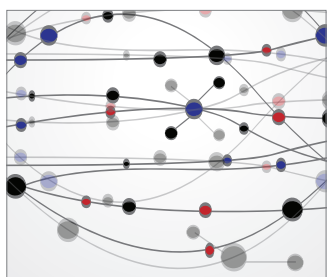

The Scientific World Journal
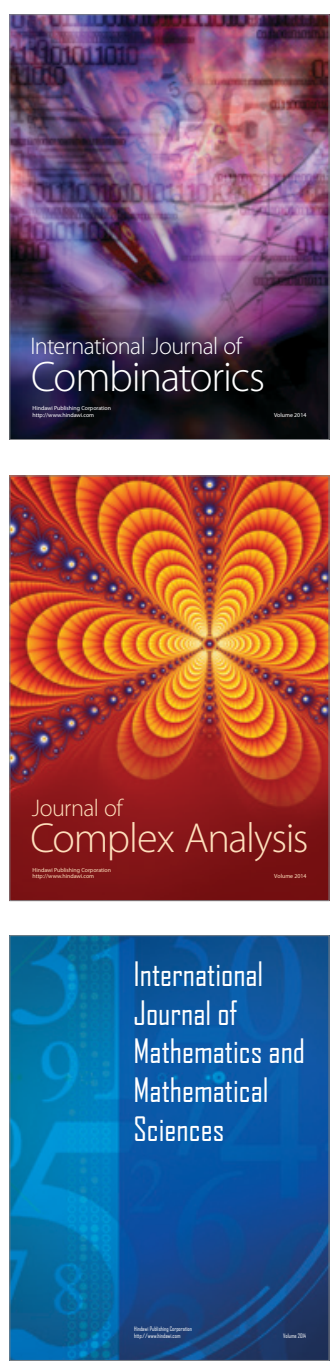
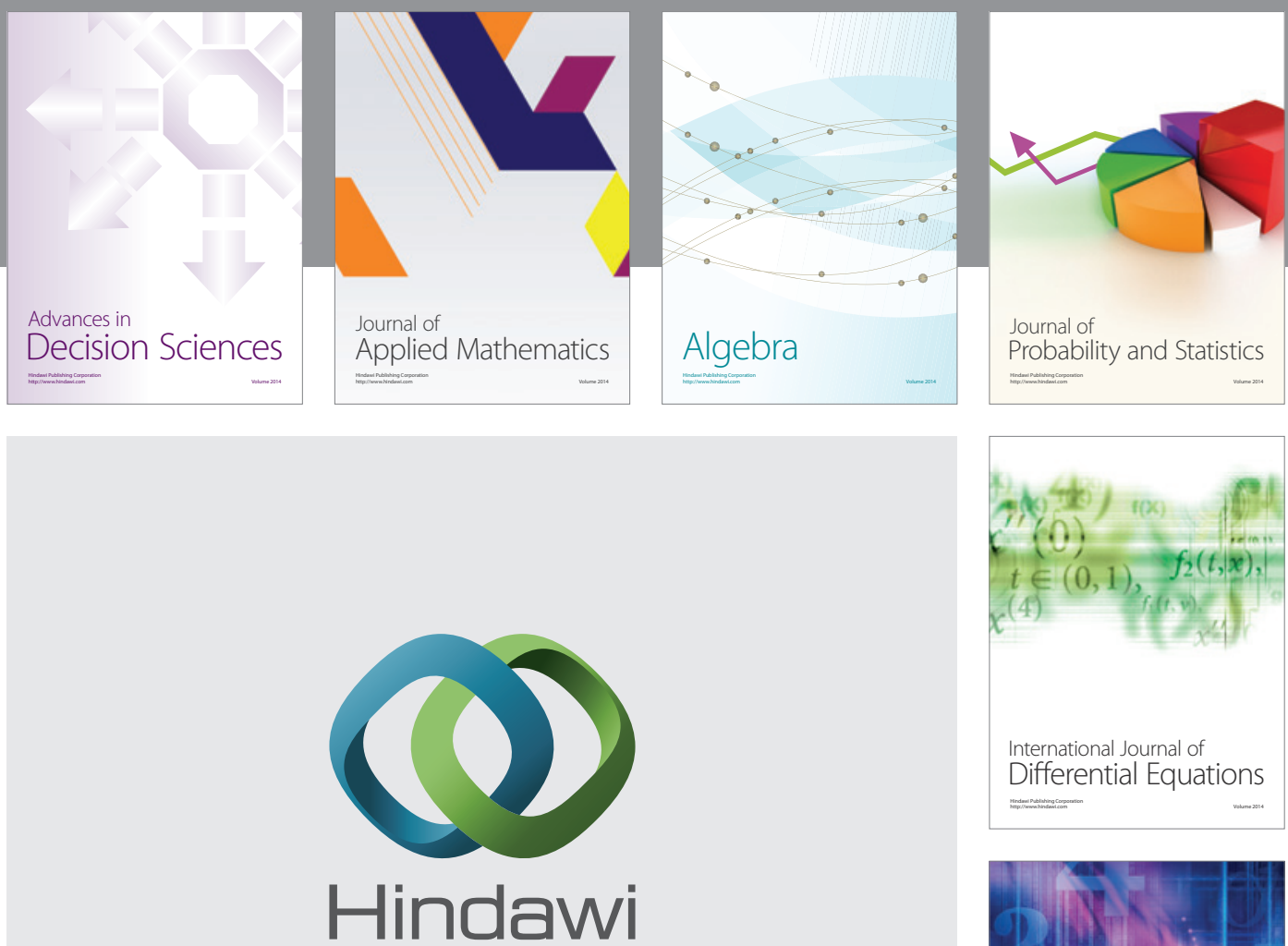

Submit your manuscripts at http://www.hindawi.com
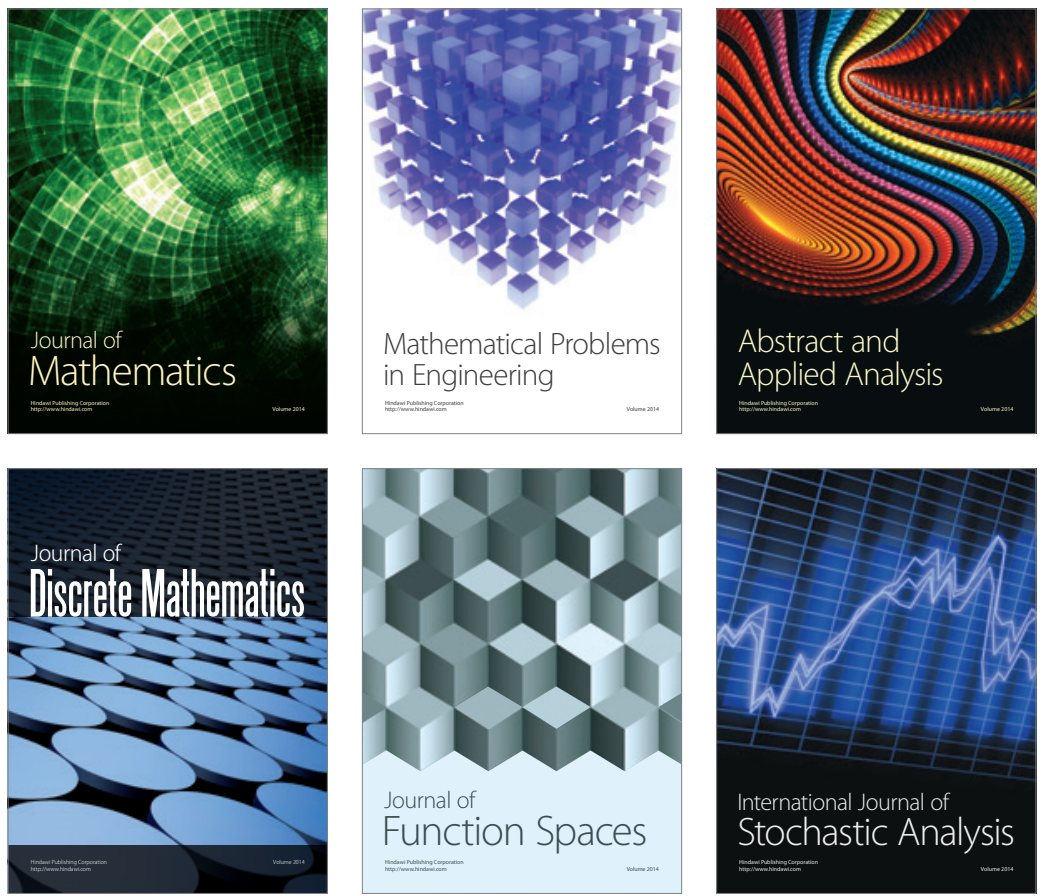

Journal of

Function Spaces

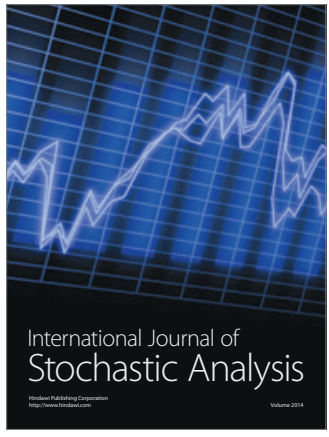

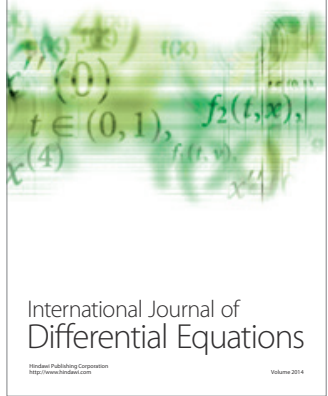
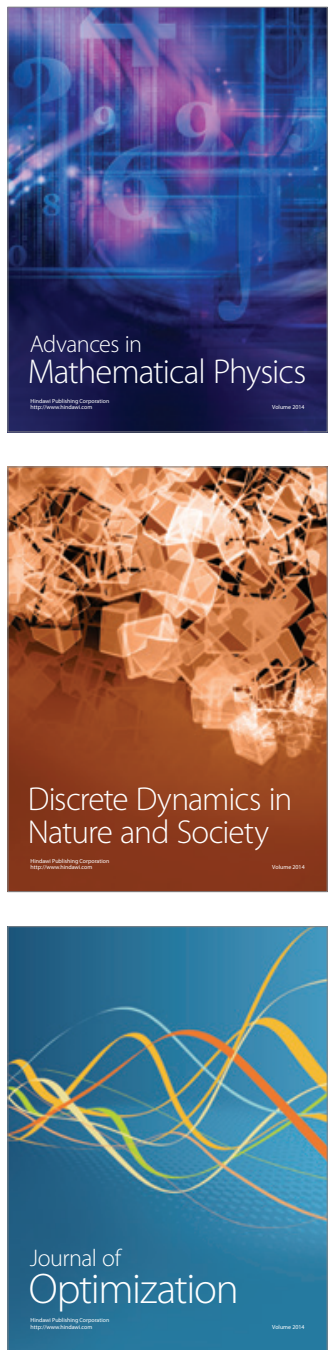